\title{
Ejercicio físico en mujeres con cáncer de mama
}

\author{
$M^{\text {a }}$ TERESA MOROS ${ }^{1}$, MERCEDES RUIDIAZ $^{1}$, ANA CABALLERO ${ }^{1}$, \\ ENRIQUE SERRANO ${ }^{1}$, VÍCTOR MARTÍNEZ ${ }^{\text {a }}$, ALEJANDRO TRES ${ }^{1,2}$
}

\section{Effects of an exercise training program on the quality of life of women with breast cancer on chemotherapy}

Background: Exercise may reduce anxiety and depression associated to the diagnosis and treatment of cancer. Aim: To assess the effects of a physical training program during chemotherapy among women with breast cancer. Patients and Methods: Twenty two women aged $49 \pm 7$ years with breast cancer voluntarily agreed to take part in the study, after surgical treatment. Functional capacity (Karnofsky Performance Status), psychological status (General Health Questionnaire, GHQ) and quality of life (EORTC QLQ-C30) were evaluated at baseline and at the end of the study. Before beginning with adjuvant chemotherapy, ten women were randomly assigned to a program of physical exercise and seven to a control group. The program lasted 18 to 22 weeks, depending on the duration of chemotherapy. Results: Five women were lost from follow up. Before starting chemotherapy, $41 \%$ of women were working and all had to kit. At baseline all had a normal Karnofski score and quality of life was compromised. At the end of the study, the intervention group had an improvement of their quality of life, compared to the control group that did not experience significant changes. Conclusions: An exercise training program improves quality of life of women with breast cancer on chemotherapy.

(Rev Med Chile 2010; 138: 715-722).

Key words: Breast neoplasms; Chemotherapy, adjuvant; Exercise.

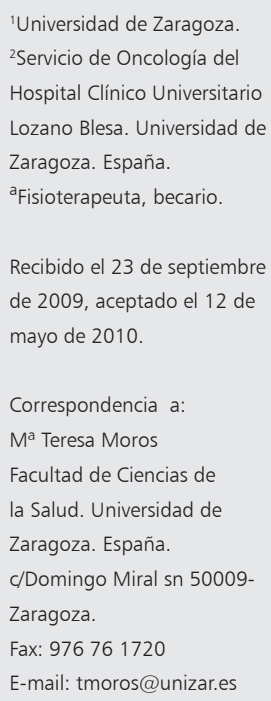

$\mathrm{E}$ 1 diagnóstico precoz y los avances que se están produciendo en la quimioterapia están posibilitando un alto porcentaje de supervivientes entre la población con cáncer de mama, pero esta supervivencia no está exenta de problemas $^{1-5}$. Tras recibir el diagnóstico de cáncer las pacientes tienden a reducir la actividad física, la cual podría estar agravando la situación de las pacientes. Dicha reducción parece guardar relación con el tipo de tratamiento al que se someten, siendo mayor en aquellas que reciben tratamiento quirúrgico asociado a quimioterapia y radioterapia frente a las que reciben sólo radioterapia o cirugía ${ }^{6}$.

Es sabido que las personas inactivas presentan fatiga, debilidad, incoordinación, reducción de las relaciones sociales, alteraciones músculo-esqueléticas, cardiovasculares y depresión; alteraciones que tienden a presentar las mujeres con cáncer de mama. Por el contrario, la práctica de ejercicio incrementa la resistencia a la fatiga, reduce la ansiedad, la depresión, mejora la capacidad funcional y el sueño, ayuda a relajarse e incrementa el trato interpersonal ${ }^{7}$.

La alta incidencia del cáncer de mama y el alto índice de supervivientes plantea la necesidad de iniciar medidas precoces. Todo lo expuesto anteriormente nos ha conducido a estudiar la situación en la que se encuentran estas pacientes 
antes de comenzar el tratamiento de quimioterapia adyuvante y a iniciar un programa de ejercicio físico durante el tratamiento de quimioterapia, y no sólo una vez finalizado el tratamiento de quimioterapia y/o radioterapia tal y como exponen diferentes autores ${ }^{8-10}$.

Los objetivos de este estudio son: 1) evaluar la capacidad funcional, la calidad de vida y estado psicológico de pacientes diagnosticadas de cáncer de mama, que han sido sometidas a tratamiento quirúrgico y que van a iniciar un tratamiento de quimioterapia; 2) valorar la influencia que tiene un programa de ejercicio físico durante todo el tratamiento de quimioterapia.

\section{Material y Método}

Estudio randomizado cuya población estudiada estuvo constituida por 22 mujeres procedentes de Oncología del "Hospital Lozano Blesa de Zaragoza”, diagnosticadas de cáncer de mama, tratadas quirúrgicamente y que van a recibir quimioterapia durante el período de estudio.

Se excluyeron aquellas pacientes que presentaban afección cardíaca y/o coronaria que contraindique la práctica de ejercicio, hipertensión, anemia severa, riesgo de fracturas, patología osteoarticular discapacitante, diabetes u otras afecciones discapacitantes.

Las pacientes que cumplieron los criterios de inclusión (edad inferior a 65 años y que no realicen habitualmente ejercicio), fueron informadas del proyecto a realizar y firmado el consentimiento para formar parte del estudio, distribuyéndose al azar en grupo de intervención $(\mathrm{GI})(\mathrm{n}=11)$ y grupo control $(\mathrm{GC})(\mathrm{n}=11)$. De las 11 pacientes del GI 10 finalizaron el estudio ( 1 paciente no fue incluida porque su adhesión al entrenamiento fue inferior al $80 \%$, por residir en zona rural y tener problemas de traslado) con una edad media de 50 años (SD: 5,5); del GC inicial, 7 pacientes realizaron las pruebas finales, con una edad media 53,14 años (SD: 6,8), (un abandono por problemas de traslado, otro por carecer de tiempo y dos pacientes por causas no justificadas). El protocolo de actuaciones cumplio las normas de Helsinki y fue aprobado por el Comité Ético de Investigación Clínica de Aragón.

Inicialmente, se ha valorado la capacidad funcional, el bienestar psíquico y la calidad de vida, tras haber recibido tratamiento quirúrgico y una semana antes de comenzar la quimioterapia. La recogida de información final se ha practicado entre los días 10 a 15 después de haber finalizado el tratamiento de quimioterapia.

\section{Valoración Funcional}

Se valoró la capacidad funcional y sus posibles cambios mediante el registro de la situación laboral y de la escala de actividad (Karnofsky Performance Status) ${ }^{11}$.

Al valorar la situación laboral, se distinguió entre situación laboral activa, baja laboral y labores de casa. En el caso de esta variable en la secuencia del registro se tuvo en cuenta no sólo la situación cuando comienza el estudio y la valoración final, además se quizo conocer la situación laboral antes de ser diagnosticada de cáncer de mama.

Se utilizó una versión simplificada del Karnofsky para que pudiera ser autoadministrada; esta escala distingue cinco niveles: $\mathrm{K}-1$ (El sujeto es capaz de desarrollar su actividad normal), K-2 (Es capaz de realizar su actividad normal pero requiere esforzarse para conseguirlo), K-3 (Sólo realiza actividades relacionadas con el cuidado de sí mismo), K-4 (Requiere asistencia para autocuidados), K-5 (Situación terminal, moribundo) ${ }^{11}$.

\section{Valoración del bienestar psicológico}

El cuestionario utilizado es el General Health Questionnaire (GHQ) ${ }^{12}$, adaptado a la población española por Lobo et $\mathrm{al}^{13}$. Consta de 4 subescalas que recogen información sobre síntomas somáticos de origen psicológico, ansiedad/angustia, disfunción social y depresión, con un total de 28 ítems (cada uno de los apartados consta de 7 ítems y la puntuación máxima por apartado es 7). Asimismo, permite obtener una valoración global del bienestar psíquico, realizándose el corte en la puntuación $6 / 7$, para determinar caso probable/ caso posible de bienestar/malestar psíquico ${ }^{13}$.

\section{Valoración de la calidad de vida}

Para evaluar la calidad de vida se utilizó The European Organization for Research and Treatment of Cancer Quality of life Questionnaire (EORTC QLQ-C30). Incluye 30 ítems (rango $0-100)$, que se reparten en valoración global (QoL), 5 escalas funcionales (funcionamiento físico, rol, emocional, social y cognitivo), 3 escalas de síntomas (fatiga, dolor y náuseas-vómitos), y 
otros ítems que valoran síntomas adicionales (disnea, pérdida de apetito, insomnio, estreñimiento, diarrea e impacto financiero). Las puntuaciones elevadas en funcionalidad indican mejor estado funcional; las puntuaciones elevadas en síntomas significan mayor gravedad de la sintomatología. El EORTC QLQ-C30 es un instrumento válido y fiable, usado en muchos estudios para evaluar las intervenciones en pacientes con cáncer ${ }^{14,15}$.

\section{Programa de ejercicio físico}

El programa de entrenamiento (realizado bajo la supervisión de los investigadores) estuvo constituido por ejercicios dinámico-aeróbicos adaptados en intensidad de forma individualizada. Se realizó una prueba de esfuerzo incremental para determinar la capacidad de esfuerzo máxima, evaluar las posibles contraindicaciones del ejercicio y determinar el esfuerzo durante el entrenamiento. La intensidad del ejercicio correspondió a 60-70\% de la frecuencia cardiaca obtenida en la prueba de esfuerzo y se controluró duante las sesiones mediante un monitor de frecuencia cardíaca (Polar HR monitor).

La duración de cada sesión fue de 60 minutos, con una frecuencia de tres sesiones/semana de entrenamiento. La duración del programa fue de 18-22 semanas dependiendo del número de ciclos de quimioterapia. Cada sesión estuvo constituida de 10 minutos de calentamiento. En la parte central de la sesión (45 minutos) se incluyeron ejercicios de fortalecimiento muscular y coordinación. Ejercicios en bicicleta estática (20 minutos), combinado con ejercicios respiratorios y de extremidades superiores (con cargas de 0,51 kilogramo en la extremidad superior del lado no intervenido), marcha sobre pista rodante (10 minutos) y trabajo de la musculatura abdominal y cintura pelviana (15 minutos). La finalización de la sesión (5 minutos) se destinaba a ejercicios de estiramientos y de relajación.

Durante el período del estudio no se ha presentado ningún incidente o efecto adverso.

\section{Estadística}

Las variables cuantitativas se describen con la media y la desviación estándar. Inicialmente se realizó un estudio descriptivo de la muestra completa y posteriormente se ha valorado la posible existencia de diferencias significativas entre el GI y el GC, utilizando el test de la U de Mann-Whitney.
Para la comparación entre las medias obtenidas entre la valoración inicial y la final se utilizó el test de Wilcoxon. La comparación de los incrementosdecrementos experimentados entre el GI y el GC, se realizó mediante el test de la U de Mann-Whitney. Los datos fueron analizados con el programa estadístico SPSS versión 14.0.

\section{Resultados}

La muestra inicial total fue de 22 pacientes. Las características demográficas y clínicas de las pacientes se presentan en la Tabla 1. Antes de recibir el diagnóstico de cáncer de mama el 40,9\% de la muestra se encontraba laboralmente activa, el resto de la población estudiada desempeñan labores del hogar. Después de recibir el diagnóstico, el 100\% de las mujeres laboralmente activas pasan a una situación de baja laboral, antes de comenzar el tratamiento, y por tanto del programa de ejercicio. Inicialmente, la capacidad funcional está conservada (Karnofsky Performance Status); todas las pacientes, excepto una, están en K-1.

El General Health Questionnaire (GHQ) pone en evidencia que las pacientes presentan manifestaciones en las cuatro áreas valoradas, siendo la manifestación de ansiedad/angustia la más relevante (Tabla 2 ).

La calidad de vida (valoración global -QoL-) se ve afectada notablemente (Tabla 3 ). Dentro de los ítems funcionales, la función emocional es la más afectada. Los síntomas dolor e insomnio son los más relevantes, seguido de la presencia de fatiga (Tabla 3$)(0=$ no en absoluto, $100=$ máxima intensidad).

La comparación inicial de las variables estudiadas, una vez randomizada la muestra grupo de intervención/grupo control, no muestran diferencias significativas.

\section{Comparación de resultados antes y después del período de estudio}

La capacidad funcional (Karnofsky Performance Status) experimenta un ligero empeoramiento en ambos grupos después del tratamiento quimioterapia/ejercicio. Nueve de las 10 pacientes del grupo de intervención pasan del nivel K-1 de Karnofsky a la valoración K-2, y sólo una paciente de este grupo se mantiene en K-1. En el grupo control, cinco de las pacientes pasan de K-1 a K-2, una 
Tabla 1. Características demográficas y clínicas $(\mathbf{n}=\mathbf{2 2})$

\begin{tabular}{|c|c|c|c|c|c|c|}
\hline & $\begin{array}{l}\text { Edad } \\
\text { (años) }\end{array}$ & IMC & Estadío & Cirugía & Quimioterapia & Ciclos \\
\hline \multicolumn{7}{|c|}{$\begin{array}{l}\text { Pacientes } \\
\text { G. Intervención }\end{array}$} \\
\hline A & 44 & 29,24 & I & MP -DA & FAC & 6 \\
\hline B & 41 & 24,46 & $\| A$ & $M P-B L$ & TxT + carboplatin + Herceptin & 6 \\
\hline C & 48 & 27,22 & $\| A$ & MT & ECx4-Txtx4 & 8 \\
\hline D & 54 & 27,41 & IIIA & $M P-B L$ & ETx4-Xeloda + adriamicina,x4 & 8 \\
\hline $\mathrm{E}$ & 51 & 26,29 & 1 & $M P-B L$ & FAC & 6 \\
\hline $\mathrm{F}$ & 57 & 26,49 & $\mathrm{IIB}$ & MT & ECx4-TxTx4 & 8 \\
\hline G & 54 & 23,80 & $\| A$ & MP -DA & ECx4-TxTx4 & 8 \\
\hline $\mathrm{H}$ & 54 & 27,27 & $\| A$ & $M P-B L$ & FACx4-Tx1x8 & 12 \\
\hline I & 42 & 21,94 & $\| A$ & $M P-B L$ & FACx4-Tx1x8 & 12 \\
\hline J & 54 & 24,13 & $\| \mathrm{A}$ & MT -DA & FAC & 6 \\
\hline \multicolumn{7}{|c|}{$\begin{array}{l}\text { Pacientes } \\
\text { G. Control }\end{array}$} \\
\hline K & 50 & 28,69 & IIB & MT -DA & FAC & 6 \\
\hline L & 47 & 22,89 & $\| A$ & MT & FACx4-Tx1x8 & 12 \\
\hline M & 62 & 36,44 & $\| A$ & MP -BL & FAC & 6 \\
\hline $\mathrm{N}$ & 64 & 31,01 & $\| A$ & MP -BL & ECx4-TxT4 & 8 \\
\hline $\mathrm{O}$ & 49 & 26,64 & IIIA & MT -DA & TEC & 6 \\
\hline$P$ & 51 & 41,12 & $\| A$ & $M P-B L$ & TEC & 6 \\
\hline Q & 49 & 28,89 & $\| A$ & $M P-B L$ & FACx4-Tx1x8 & 12 \\
\hline \multicolumn{7}{|c|}{ Abandonos } \\
\hline $\mathrm{R}$ & 49 & 29,73 & $\| A$ & $M P-B L$ & $A C$ & 6 \\
\hline S & 42 & 28,07 & $\| \mathrm{B}$ & MP -BL & TEC & 6 \\
\hline T & 61 & 24,68 & IIB & MP -DA & TEC & 6 \\
\hline U & 38 & 21,09 & 1 & MT & $A C$ & 6 \\
\hline W & 41 & 18,08 & $\| \mathrm{B}$ & MP -BL & TEC & 6 \\
\hline
\end{tabular}

Abreviaturas: IMC (índice de masa corporal); DA (disección axilar); BL (biopsia linfonodo centinela); MP (mastectomía parcial); MP (mastectomía total); FAC (fluorouracilo, adriamicina, ciclofosfamida); TxT (Taxotere); EC (epirubicina, ciclofosfamida); ET (epirubicina, taxol); AC (adriamicina, ciclofosfamida); TEC (Taxotere, epirubicina, ciclofosfamida).

Tabla 2. Evaluación psicológica antes de la quimioterapia adyuvante $(\mathbf{n}=\mathbf{2 2})$. General Health Questionaire-GHQ

\begin{tabular}{|lcc|}
\hline & Media $\pm \mathbf{D E}$ & (puntación máxima 7) \\
\hline Síntomas somáticos de origen psicológico & $1,22 \pm 1,77$ & $"$ \\
\hline Ansiedad/angustia & $2,77 \pm 2,02$ & $"$ \\
\hline Disfunción social & $1,22+1,26$ & " \\
Depresión & $0,63 \pm 1,17$ & (puntación máxima 28) \\
\hline Valoración global & $5,86 \pm 3,53$ & \\
\hline
\end{tabular}

$\mathrm{DE}=$ desviación estándar. 
Ejercicio físico en mujeres con cáncer de mama - $\mathrm{M}^{\mathrm{a}} \mathrm{T}$. Moros et al

Tabla 3. Evaluación de la calidad de vida antes de la quimioterapia adyuvante $(n=22)$

\begin{tabular}{|lc|}
\hline QOL-C30 & Media \pm DE \\
QoL & $69,32+16,34$ \\
Escala funcional & \\
Función física & $90,90 \pm 9,09$ \\
Role función & $81,81 \pm 18,48$ \\
Función emocional & $68,93 \pm 17,28$ \\
Función cognitiva & $90,15 \pm 22,80$ \\
Función social & $85,60 \pm 18,03$ \\
Escala de síntomas & $19,69 \pm 14,10$ \\
Fatiga & $0,00 \pm 0,00$ \\
Náuseas-vómitos & $25,75 \pm 18,34$ \\
Dolor & $1,51 \pm 7,10$ \\
Disnea & $31,81 \pm 19,18$ \\
Insomnio & $7,57 \pm 14,29$ \\
Bajo apetito & $24,24 \pm 37,34$ \\
Constipación & $1,51 \pm 7,10$ \\
Diarrea & $12,12 \pm 19,36$ \\
Dificultades financieras & \\
\hline
\end{tabular}

The European Organization for Research and Treatment of Cancer Quality of Life Questionaire EORTC QLQ- C30. (Rango 0-100). paciente lo hace a K-3 y la otra paciente permanece en K-1. Tras finalizar la quimioterapia y programa de ejercicio, el GI presenta un deterioro, no significativo, de su bienestar psíquico global (Tabla 4). El GC muestra un empeoramiento significativo en síntomas somáticos de origen psicológico ( $\mathrm{p}$ $<0,05)$ y también en la valoración global $(\mathrm{p}<$ $0,05)$ (Tabla 4).

La calidad de vida (QoL) mejora significativamente en el GI después del período de ejercicio ( $\mathrm{p}$ $=0,005$ ), hecho que no se observa en el GC (Tabla 5). La percepción de la fatiga se ve incrementada en el GI durante el tratamiento quimioterápico/ ejercicio, presentando al finalizar el estudio una intensidad en su percepción similar a la del grupo control.

La comparación de los incrementos-decrementos de las variables estudiadas entre los grupos de estudio, muestra diferencias sólo en la calidad de vida $(\mathrm{QoL})(\mathrm{p}=0,010)$.

\section{Discusión}

El conocimiento de sufrir un cáncer de mama, la incertidumbre que experimentan, tanto por la evolución de su enfermedad como por su supervivencia y el sometimiento a un tratamiento quirúrgico, influyen negativamente sobre la población estudiada tal y como quedan reflejados en los resultados.

La capacidad funcional se ve afectada por el

Tabla 4. Estado psicológico antes/después del período de estudio. General Health Questionaire-GHQ

\begin{tabular}{|c|c|c|c|c|c|c|}
\hline & \multicolumn{3}{|c|}{ G. Intervención $(n=10)$} & \multicolumn{3}{|c|}{ G. Control $(n=7)$} \\
\hline & $\begin{array}{c}\text { Pre } \\
\text { Media } \pm \text { DE }\end{array}$ & $\begin{array}{c}\text { Post } \\
\text { Media } \pm \text { DE }\end{array}$ & $\mathbf{p}$ & $\begin{array}{c}\text { Pre } \\
\text { Media } \pm \text { DE }\end{array}$ & $\begin{array}{c}\text { Post } \\
\text { Media } \pm \text { DE }\end{array}$ & $\mathbf{p}$ \\
\hline $\begin{array}{l}\text { Síntomas somáticos de } \\
\text { origen psicológico }\end{array}$ & $1,50 \pm 2,1$ & $3,20 \pm 2,89$ & 0,114 & $1,00 \pm 1,52$ & $3,28 \pm 1,70$ & 0,041 \\
\hline Ansiedad/angustia & $2,90 \pm 1,66$ & $2,30 \pm 2,58$ & 0,573 & $3,28 \pm 2,92$ & $4,00 \pm 2,58$ & 0,461 \\
\hline Disfunción social & $1,40 \pm 1,50$ & $2,60 \pm 2,31$ & 0,071 & $1,00 \pm 1,00$ & $2,00 \pm 1,91$ & 0,109 \\
\hline Depresión & $0,40 \pm 1,26$ & $1,00 \pm 2,16$ & 0,059 & $0,71 \pm 1,11$ & $1,28 \pm 2,62$ & 0,414 \\
\hline Valoración global & $6,20 \pm 4,04$ & $9,10 \pm 8,39$ & 0,221 & $6,00 \pm 3,51$ & $10,57 \pm 8,05$ & 0,043 \\
\hline
\end{tabular}


Tabla 5. Calidad de vida antes/después del período de estudio

\begin{tabular}{|c|c|c|c|c|c|c|}
\hline & \multicolumn{3}{|c|}{ G. Intervención $(n=10)$} & \multicolumn{3}{|c|}{ G. Control $(n=7)$} \\
\hline & Pre & Post & & Pre & Post & \\
\hline & Media \pm DE & Media \pm DE & $\mathbf{p}$ & Media \pm DE & Media \pm DE & $\mathbf{p}$ \\
\hline \multicolumn{7}{|l|}{ QLQ-C30 } \\
\hline QoL & $65,0 \pm 0,1$ & $70,0 \pm 18,9$ & 0,005 & $72,6 \pm 8,8$ & $60,7 \pm 19,0$ & 0,080 \\
\hline \multicolumn{7}{|l|}{ Escala funcional } \\
\hline Función física & $92,0 \pm 9,3$ & $86,6 \pm 14,7$ & 0,147 & $91,4 \pm 6,3$ & $87,6 \pm 19,0$ & 0,854 \\
\hline Rol función & $83,3 \pm 15,7$ & $78,3 \pm 19,3$ & 0,408 & $88,0 \pm 20,8$ & $80,9 \pm 24,3$ & 1 \\
\hline Función emocional & $70,8 \pm 16,7$ & $70,0 \pm 28,1$ & 0,859 & $65,4 \pm 18,8$ & $61,9 \pm 28,8$ & 0,916 \\
\hline Función cognitiva & $90,0 \pm 14,0$ & $83,3 \pm 17,5$ & 0,245 & $100,0 \pm 0,0$ & $88,0 \pm 24,9$ & 0,180 \\
\hline Función social & $81,6 \pm 19,9$ & $76,6 \pm 22,4$ & 0,436 & $90,4 \pm 13,1$ & $88,0 \pm 20,8$ & 0,785 \\
\hline \multicolumn{7}{|l|}{ Escala de síntomas } \\
\hline Fatiga & $15,5 \pm 10,7$ & $31,1 \pm 24,4$ & 0,020 & $19,0 \pm 12,3$ & $30,1 \pm 18,9$ & 0,666 \\
\hline Náuseas/vómitos & $0,0 \pm 0,0$ & $5,0 \pm 11,2$ & -- & $0,0 \pm 0,0$ & $9,5 \pm 13,1$ & -- \\
\hline Dolor & $25,0 \pm 14,1$ & $21,6 \pm 26,1$ & 0,951 & $16,6 \pm 23,5$ & $16,6 \pm 16,6$ & 0,102 \\
\hline Disnea & $3,3 \pm 10,5$ & $6,6 \pm 14,0$ & 0,564 & $0,0 \pm 0,0$ & $19,0 \pm 26,2$ & -- \\
\hline Insomnio & $30,0 \pm 24,5$ & $23,3 \pm 35,3$ & 0,763 & $33,3 \pm 0,0$ & $14,2 \pm 26,2$ & 0,102 \\
\hline Apetito & $10,0 \pm 16,1$ & $10,0 \pm 16,1$ & 1 & $0,0 \pm 0,0$ & $4,7 \pm 12,5$ & 0,317 \\
\hline Constipación & $13,3 \pm 32,2$ & $23,3 \pm 35,3$ & 0,680 & $33,3 \pm 38,4$ & $28,5 \pm 40,4$ & 0,785 \\
\hline Diarrea & $0,0 \pm 0,0$ & $6,6 \pm 14,0$ & 0,157 & $0,0 \pm 0,0$ & $9,5 \pm 16,2$ & 0,157 \\
\hline Dificultad financiera & $10,0 \pm 16,1$ & $20,0 \pm 28,1$ & 0,257 & $19,0 \pm 26,2$ & $33,3 \pm 27,2$ & 0,083 \\
\hline
\end{tabular}

The European Organization for Research and Treatment of Cancer Quality of Life Questionaire EORTC QLQ- C30

tratamiento quirúrgico, sin embargo, Burnham et $\mathrm{al}^{16}$, exponen que los cambios que experimentan los pacientes con diferentes tipos de cáncer se pueden ver, en ocasiones, influenciados positivamente por el mero paso del tiempo, con una mejora de la capacidad funcional (Karnofsky). No parece ocurrir lo mismo con la calidad de vida que se va deteriorando, durante y después del tratamiento del cáncer de mama, y la variable -tiempo-no parece influir positivamente sobre el QoL tras finalizar la terapia ${ }^{3}$. Sin embargo, Herrero et $\mathrm{al}^{17}$, exponen que no existe una correlación entre el resultado de la valoración global en el QoL y el tiempo posttratamiento si el registro se practica tardíamente (2-5 años de finalizado el tratamiento).

Entre las diferentes terapias para combatir las alteraciones psicológicas experimentadas por los pacientes cancerosos se incluye la práctica de ejercicio físico postratamiento ${ }^{18,19}$. En el período de estudio comprobamos un deterioro psicológico, aunque se obtiene una discreta tendencia a la mejoría de la ansiedad/angustia en el grupo de intervención frente al grupo control que presenta un incremento significativo de su malestar psíquico en la valoración global. Mock et $\mathrm{al}^{20}$, también consiguen reducir la ansiedad iniciando el programa de entrenamiento durante la radioterapia. Midtggard et $\mathrm{al}^{21}$, obtienen una mejora significativa en el estado de ansiedad y depresión, pero exponen que el ejercicio es sobre todo beneficioso si se presentan unos bajos o moderados niveles de psicomorbilidad. Nuestros resultados concuerdan con estos autores que, independientemente del tipo o fase de tratamiento aplicado, la realización de ejercicio determina efectos favorables en las manifestaciones de ansiedad/angustia.

Diversos autores exponen efectos positivos del entrenamiento sobre la calidad de vida tras 
Ejercicio físico en mujeres con cáncer de mama - $\mathrm{M}^{\mathrm{a}} \mathrm{T}$. Moros et al

finalizar el tratamiento $(0,8$ a 21 años postquimioterapia $)^{8,19,22,23}$. En este estudio se obtiene una mejora significativa en el Global Health Status (QoL), hecho que no ocurre en el grupo control. Sin embargo, sí que se presenta un aumento de la intensidad de la fatiga resultando similar en el grupo de intervención y en el de control. Se desconocen los factores y mecanismos determinantes de la fatiga; se puede hacer referencia al efecto de la quimioterapia sobre las fibras musculares, la anemia, el malestar psíquico, el insomnio, o la inactividad ${ }^{24}$. Se han observado cambios metabóli$\cos$, en la ultraestructura y en la función muscular ${ }^{23}$ durante la quimioterapia por el efecto miotóxico de las sustancias utilizadas (disminución de la masa miofibrilar, y del número/tamaño de mitocondrias), reducción de la microcirculación e incluso rabdomiolisis, $y$ estos cambios provocan reducción de la fuerza, sensación de debilidad y deterioro funcional ${ }^{24}$. Neuromoduladores como el ammonia o citokinas segregadas por las células inmunes pueden actuar sobre el sistema nervioso alterando la percepción y reduciendo la capacidad de actividad ${ }^{25}$. Es posible que debido a todo ello las pacientes con quimioterapia no mejoren su percepción de fatiga.

El insomnio es el síntoma con puntuación más alta en las pacientes de este estudio. Payne et $\mathrm{al}^{26}$, realizan un programa de entrenamiento post-quimioterapia obteniendo una mejora en la calidad del sueño. En este estudio se consigue reducir levemente el insomnio; posiblemente el hecho de liberarse de la quimioterapia sea el motivo de la discreta mejoría de este síntoma, ya que observamos la misma respuesta en el grupo de control.

El dolor está presente en la muestra estudiada y el ejercicio tiende a reducirlo ligeramente. CooK et $\mathrm{al}^{27}$, aconsejan la actividad física como medio de atenuar dicho síntoma en diversas afecciones. Los mecanismos responsables de este efecto no están aclarados, aunque entre ellos se hace referencia al aumento de secreción de opiáceos endógenos.

Son escasos los autores que inician el programa de ejercicio durante la quimioterapia. Campbell et $\mathrm{al}^{9}$, incluyen en su muestra un escaso número de pacientes $(\mathrm{n}=3)$ con cáncer de mama sometidas a quimioterapia (iniciando el entrenamiento entre el 2-3 $3^{\text {er }}$ ciclo), y asocian para completar la muestra $(n=12)$, otras pacientes bajo tratamiento radioterápico. Estos autores refieren mejoras en la valoración global (QoL) y en la función física, pero tampoco obtienen una reducción de la fatiga tras 12 semanas de entrenamiento. Otros autores ${ }^{28}$ también inician el entrenamiento durante la terapia, pero solamente el $30 \%$ recibe quimioterapia. En estos estudios los resultados están expresados en valores medios de todo el grupo experimental por lo que no se tiene un conocimiento real de las variaciones ocurridas durante la quimioterapia.

Un motivo de preocupación es la adhesión al ejercicio $^{28,29}$. Una de las limitaciones en este estudio podía ser el encontrarnos con un alto grado de abandono, sin embargo, hemos obtenido una buena colaboración del GI; el grado de colaboración del GC ha sido menor.

\section{Referencias}

1. Wilmoth MC, Coleman EA, Smith SC, Davis C. Fatigue, weight gain, and altered sexuality in patients with breast cancer: exploration of a symptom cluster. Oncol Nurs Forum 2004; 31: 1069-75.

2. Brain K, Williams B, Iredale R, France L, Gray J. Psychological distress in men with breast cancer. J Clin Oncol 2006; 24: 95-101.

3. Montazeri A. Health-related quality of life in breast cancer patients: a bibliographic review of the literature from 1974 to 2007. J Exp Clin Cancer Res 2008; 27: 32.

4. Byar KL, Berger AM, Bakken SL, Cetak MA. Impact of adjuvant breast cancer chemotherapy on fatigue, other symptoms, and quality of life. Oncol Nurs Forum 2006; 33: 18-26.

5. Irwin ML, Crumley D, McTiernan A, Bernstein L, Baumgartner R, Gilliland FD, et al. Physical activity levels before and after a diagnosis of breast carcinoma: the Health, Eating, Activity, and lifestyle (HEAL) study. Cancer 2003; 97: 1746-57.

6. Valenti M, Porzio G, Aielli F, Verna L, Cannita K, Manno $\mathrm{R}$, et al. Physical exercise and quality of life in breast cancer survivors. Int J Med Sci 2008; 5: 24-8.

7. Warburton DE, Nicol CW, Bredin SS. Health benefits of physical activity: the evidence. CMAJ 2006;174: 801-9. Review.

8. Cheema BS, Gaul CA. Full-body exercise training improves fitness and quality of life in survivors of breast cancer. J Strength Cond Res 2006; 20: 14-21.

9. Campbell A, Mutrie N, White F, McGuire F, Kearney N. A pilot study of a supervised group exercise programme as a rehabilitation treatment for women with breast cancer receiving adyuvant treatment. Eur J of Oncol Nurs 2005; 9: 56-63. 
10. Matheus CE, Wilcox S, Hanby CL, Ananian Ch, Heiney SP, Gebretsadik T, et al. Evaluation of a 12-week homebase walking intervention for breast cancer survivors. Support Care Cancer 2007; 15: 2003-11.

11. Karnofsky DA, Burchenal JH. The clinical evaluation of chemotherapeutic agents in cancer. N Eng J Med 1949; 304: 309-14.

12. Golberg DP, Hillier VF. A scale version of the general health questionnaire. Psychol Med 1979; 9: 139-45.

13. Lobo A, Pérez JM, Artal J. Validity of the scale version of the general health questionnaire (GHQ-28) in a Spanish population. Psichol Med 1986; 16: 135-40.

14. Fayers PM, AAronson NK, Bjodal D, Curran M, Groenvol D. On behalf of the EORTC Quality of Life Group. The EORTC QLQ-C30 Scoring Manual (3 ${ }^{\text {rd }}$ ed). Brussels: European Organisation for Research and Treatment of Cancer 2001; 1-86.

15. Aaronson NK, Ahmedzai S, Bergman B, Bullinger M, Cull A, Duez NJ. The European for research and treatment of cancer QLQ-C30: a quality-of-life instrument for use in international clinical trials in oncology. J Natl Cancer Inst 1993; 85: 365-76.

16. Burnham TR, Wilcox A. Effects of exercise on physiological and psychological variables in cancer survivors. Med Sci Sports Exerc 2002; 34: 1863.

17. Herrero F, Balmer J, San Juan AF, Foster C, Fleck SJ, Pérez $\mathrm{M}$, et al. Is cardiorespiratory fitness related to quality of life in survivors of breast cancer? J Strength Cond Res 2006; 20: 535-40.

18. Peled R, Carmil D, Siboni-Samocha O, Shoham-Vardi I. Breast cancer, psychological distress and life events among young women. BMC Cancer 2008; 22: 245.

19. Korstjens I, Mesters I, Vander Peet E, Gijsen B, Van den Borne B. Quality of life of cancer survivors after physical and psychosocial rehabilitation. Eur J Cancer Prev 2006; 15: 541-7.

20. Mock V, Dow KH, Meares CJ, Grimm PM, Dienemann JA, Haisfield-Wolfe ME, et al. Effects of exercise on fati- gue, physical functioning, and emotional distress during radiation teraphy for breast cancer. Oncol Nurs Forum 1997; 24: 991-1000.

21. Midtggard J, Rorth M, Stelter R, Tveteras A, Andersen C, Quist M, et al. The impact of a multidimensional exercise program on self-reported anxiety and depression in cancer patients undergoing chemotherapy: a phase II study. Palliat Support Care 2005; 3: 197-208.

22. Courneya KS, Friedenreich CM, Sela RA, Quinney HA, Rhodes RE, Handman M. The group psychotherapy and home-based physical exercise (group-hope) trial in cancer survivors: physical fitness and quality of life outcomes. Psychooncology 2003; 12: 357-74.

23. Herrero F, San Juan AF, Fleck SJ, Foster C, Lucía A. Effects of detraining on the functional capacity of previously trained breast cancer survivors. Int J Sports Med 2007; 28 (3): 257-64.

24. Visovsky C. Muscle strength, body composition, and physical activity in women receiving chemotherapy for breast cancer. Integr Cancer Ther 2006; 5: 183-91.

25. Shima E, Hino M, Mayame T, Aoyama Y, Nakamae H, Yamamura R, et al. Acute rhabdomyolisis following administration of high-dose cyclophosphamide: case report. Ann Hematol 2002; 81: 55-6.

26. Payne JK, Held J, Thorpe J, Shaw H. Effect of exercise on biomarkers, fatigue, sleep disturbances, and depressive symptoms in older women with breast cancer receiving hormonal therapy. Oncol Nurs Forum 2008; 35: 635-42.

27. CooK DB, Koltyn KF. Pain and exercise. Int J Sport Psychol 2000; 31: 256-77.

28. Kim CH, Kang D, Smith B, Landers K. Cardiopulmonary responses and adherence to exercise in women newly diagnosed with breast cancer undergoing adjuvant therapy. Cancer Nurs 2006; 29: 156-64.

29. Courneya KS, Segal RJ, Gelmon K, Reid RD, Mackey JR, Friedenreich CM, et al. Predictors of supervised exercise adherence during breast cancer chemotherapy. Med Sci Sports Exerc 2008; 40: 1180-7. 